\title{
Analysis of Twitter Unfollow: How often Do People Unfollow in Twitter and Why?
}

\author{
Sue Moon \\ Computer Science Department, KAIST \\ 291 Daehak-ro, Yuseong-gu, Daejeon, Korea \\ sbmoon@kaist. edu
}

\begin{abstract}
Unfollow in Twitter offers a unique opportunity to researchers to study the dissolution of relationship. We collected daily snapshots of follow relationship of 1.2 million Korean-speaking users for 51 days and their all tweets. From careful statistical analysis, we confirm that unfollow is prevalent and irrelevant to the volume of interaction. We find that other factors such as link reciprocity, tweet burstiness and informativeness are crucial for unfollow decision. We conduct interview with 22 users to supplement the results and figure out motivations behind unfollow behavior. From those quantitative and qualitative research we draw significant implications in both theory and practice. Then we use a multiple logistic regression model to analyze the impacts of the structural and interactional properties on unfollow in Twitter. Our model with 42 dependent variables demonstrates that both structural and interactional properties are important to explain the unfollow behavior. Our findings are consistent with previous literature about multiple dimensions of tie strength in sociology but also add unique aspects of unfollow decision that people appreciate receiving attention rather than giving.
\end{abstract}

